\title{
Appraisal on Applications of Nanorobots
}

\author{
RajaniReddy .R ${ }^{(1)}$, Vidya shree.R ${ }^{(2)}$, Asst Prof Rajeshwari ${ }^{(3)}$ \\ ${ }^{I}$ (Department of Information Science, New Horizon college of Engineering, India) \\ ${ }^{2}$ (Department of Information Science, New Horizon college of Engineering, India) \\ ${ }^{3}$ (Department of Information Science, New Horizon college of Engineering, India)
}

\begin{abstract}
Nanotech is the manipulation of the matter on atomic and molecular scale. Nanorobots being an emerging technology used precisely in machines or robots to perform controlled manipulation of objects with nanometre scale dimensions. The Nanorobot components are at a scale of nanometre $\left(10^{-9}\right)$ ranging from 0.1 10 micrometer.This paper presents a comprehensive survey on novel medical applications by Nanorobots. Recent works on the Nanorobots such as preventing ailments, treating cancer, alleviating pain and progressing human health has been described specifically. The aspect of this paper is on the Nanorobots medical applications and challenges to control its movement in human body.
\end{abstract}

Keywords: Nanodentistry, Nanomachine, Nanometer, Nanotech, Nanomedicine.

\section{Introduction}

Nanotechnology deals with the measured function of objects with nanometer scale dimensions. Over the period of time Nanotechnology era is rapidly upcoming which was unheard two decades ago. The dimension of the Nanorobot is of the order $10^{(-9)}$ as shown in the Fig.1. All orders of the human life will be impacted by progress in nanotechnology in the near future.

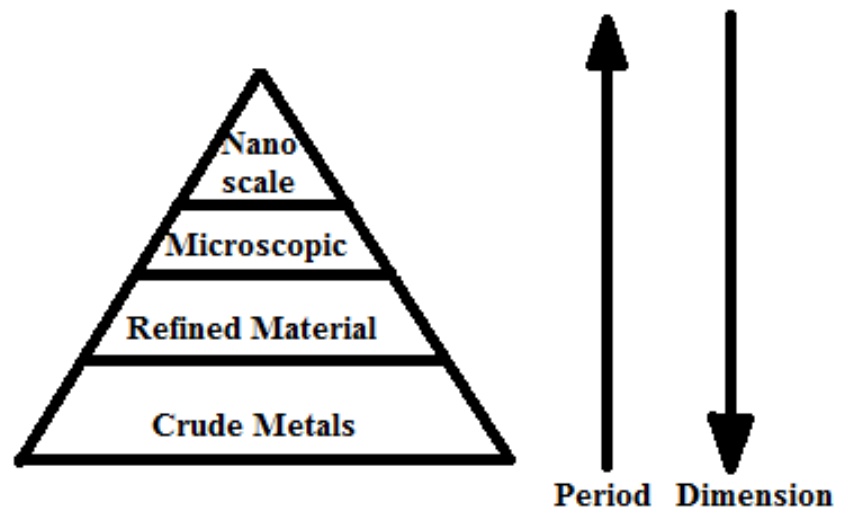

Fig.1.Human Technical Advancement --from the Stone Age to the 21st Century, the level of precision that

Humans have been able to achieve has increased with the complexity of their tools and materials.

This increasing interest in this field gives emergence to a new field called Nanomedicine a discipline \& technology of diagnosing, treating \& avoiding diseases and safeguard \& improving human health, using nanoscale structured materials called as Nanorobots. Nano in Greek means 'dwarf'and Robots are programmable machines. A Nanorobot is a specialized nanomachine. It has dimensions on the order of nanometres, typically of 0.5 to 3 microns large with $1-100 \mathrm{~nm}$. The progression of Nanorobots is a systematic development that could permit real time in vivo projection for application in a wide range of biomedical issues. Recently, Nanorobots have been made to function as a next cohort pills which is a Nano-shaped capsule, carrying the required curative material for the targeted area.

A main barrier facing nanotechnology today is the lack of effective processes for building the nanoscale structures required by the envision applications required to follow and its procedures both in quality and quantity in 3Dimension biological system.

\section{Potential Applications}

Potential applications for Nanorobotics in medicine include Nanodentistry, drug-delivery for cancer, cleaning wounds, demyelination treatment, breaking kidney stones, DNANanorobotics monitoring of diabetes and health care etc.This paper describes a number of recently reported Nanorobots development activities in medical field and the current status. They are explained below, 


\subsection{Treatment Of Cancer}

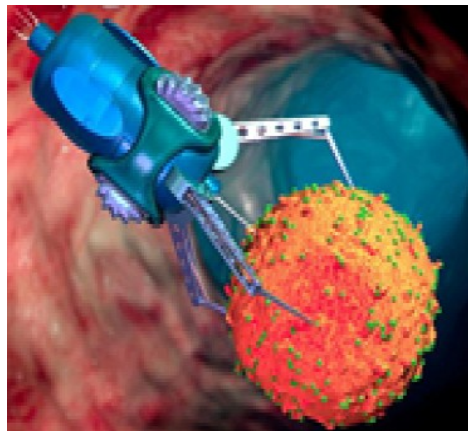

Fig2.Nanorobots attacking the cancer cells.

The above picture shows the Nanorobot attacking the cancer cell. According to W.H.O the main cause for deaths now-a-days is cancer. The traditional methods used in the treatment of cancer are Radio therapy and Chemo therapy. But the patient undergoing this treatment has to bear lots of pain and side effects like fatigue, hair loss, weight loss etc...So, to avoid this researchers have now come up with an idea of using Nanorobots in treatment of cancer. So by using Nanorobots a cancer patient can be cured completely without any side effects. This work presents an innovative approach to build, control, and monitoring medical Nanorobot in diagnosis of cancer for its early stage before metastasis. The proposed work uses biomedical flows, E-cadhering signal analyses, and RF CMOS, allied with mobile phones, for the Nanorobot architecture development.

Here in this method the patient will be given an injection which contains the Nanorobots. Three micron is a top limit of any Nanorobot because s Nanorobots whose size is larger than this will block capillary flow.

A higher gradient of signal intensity of E-cadherin is used as chemical parameter which will search out only for the cancer cells and destroy them. The other healthy cells will not be affected at all. A Nanorobot can effectively use chemical signals and targeted proteins to improve treatment intervention time to identify tumour cells [2]. A single tumour cell in a small venal is used as a target for medical diagnosis through tumour identification. The patient undergoing this treatment need not know about the Nanorobots but will notice the change in his health

This same approach presented here could widely benefit likewise other diseases and medical problems, in areas such as cardiology, diabetes, and cell therapy. The use of nanoelectronics is enabling manufacturing of nanoscale devices such as sensors and actuators for data transmission and assembly of transducers [1]. The Nanorobots use a practical methodology for a collective action with a distributed sensing in the combat of cancer. Thus, effective pervasive analyses can eliminate the risk to detect cancer only in advanced stages. Using medical Nanorobotics, integrated nanoelectronics, and wireless system, as an optimal solution for cancer detection and diagnosis, can help save lives through more precise cellular malignant tissues identification [3].Fig 1 shows Nanorobot attacking the cancer cell.

\subsection{Nanodentistry}

Nanodiagnostic devices are able to be used for early infection recognition at the cellular and molecular levels. From an in vivo perspective, nanodevices might be placed in into the body to recognize the timely occurrence of a disease in order to recognize and enumerate toxic molecules, tumour cells and so forth(Freitas, 2000;Lampton,1995). This indicator has being deliberated with the help of atomic force microscopy ,which utilize nanoparticles [4][5]. The pasture of dentalnanomedicine involve the usage of three modules of molecular arrangement they are nanomaterials, non-biological nanoparticles, materials and mechanism based on biotechnology and non-biological instruments including Nanorobotics needed for diagnosis andtherapy purposes. 


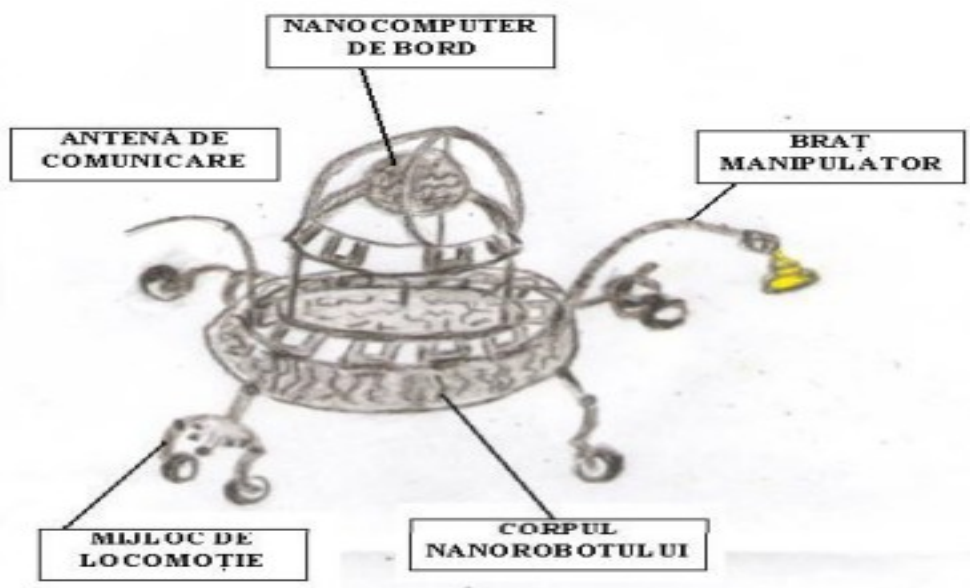

Fig.3 Dental Nanorobot.

The dental Nanorobot shown in the Fig.3 consists of nanocomputers on plank that will store and perform designed missions. It will obtain and route signals and exterior stimuli and will commune with nanocomputers which would react to exterior control and examine devices and will possess the contextual information such that correct functioning of the nanomechanical devices occurs. The structure of Nanorobots is shown in Fig 3. The various healing nature of Nanodentistry are,

2.2.1 Healing of oral cancer: Nanorobots get in touch through surface of the cap can be able to attain the soft tissue through the gingival sulks, lamina propria as shown in the Fig3.1 [4].

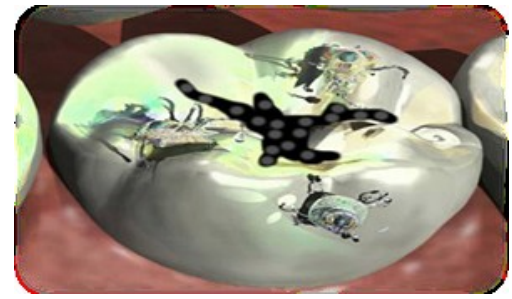

Fig3.1 Nanorobots heal oral cancer.

2.1.2 Orthodontic: The robots permit easy tooth rotating and vertical repositioning, and quick tissue restore .A stainless steel cable which needs Nanorobot that unite ultra-high strength by means of deformability, rust resistance, and surface end.

2.2.3 Keeping a perfect oral hygiene: It is done by the using dentifrobots, a major part in reducing the cavities and periodontal infection. It could be done by using them in toothpaste and mouthwash. Dentifrobots have the ability to reach places where toothbrush can't. It can recognize and destroy pathogen germs.

Problems associated with dental Nanorobots: The doable vanishing of human beings if the Nanorobots cannot be removed from the human body, however there may be disappearance of biosphere if these Nanorobots will grow towards the ability of constant auto duplication which leads to emergence of clusters overriding the living creature on Earth, leaving behind a futile grey mass [6].

\subsection{Dna Nanorobotics}

Deoxyribo nucleic acid is a particle that encodes the genetic instructions required for development and execution of living organisms and many viruses. Through scheming a DNA series just accurate, scientists are able to obtain the resultant floppy thread to join to itself and turn into any shape that they need. The resultant is DNA origami that creates 3D arrangement which is only nanometres in size. Nano sized entities formed of DNA are able to perform the same kind of logic operation as a silicon-based computer has been introduced into a living animal [7]. DNA Nanomachines which control conformation according to their environment.DNA induced by changes in salt concentration i.e., sodium and magnesium ions was achieved with a short piece of DNA and used conformational change linking $Z$ and Bforms of this DNA segment to change the position the two DX tiles. When the linking section is in the B form, the DX strips are on the similar surface of the linking section, whereas in the $\mathrm{Z}$ form they change to converse sides. This conformational switching activities is explained using FRET experiments with nanobots we can reassess that DNA nanomachine are highly specialized, they are used for performing specific task like carrying cargo, altering shape as shown in Fig4 


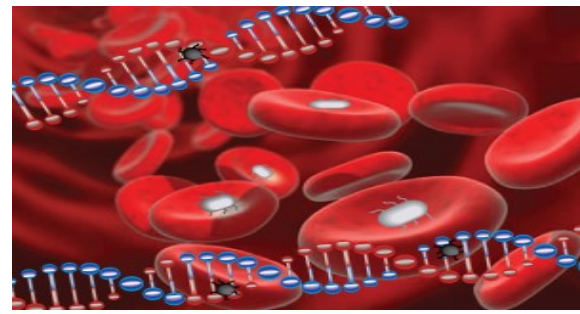

Fig 4. Nanorobot altering shape of DNA.

The input is programmed as a set of hairpins on the rambler which are consecutively processed by different DNAzymes of the machine The DNAzymes which presently bind the rambler indicates the state of the automaton thus leading to programmable DNA Nanorobots.Scientists have designed Nanorobots that imitate cockroaches and still surrounded electronics inorder to distant control the bugs. Researchers say that it must be possible to extent up the computing authority in the cockroach to that of an 8-bit computer [7] and if you tug this phenomenon of nanobots to respond to each other's expansion, then it is possible to convert organic bits into an improved biological computer [8].

Different challenges occur in effort to produce a DNA Nanorobot. The recording of domains to DNA series is made with care to evade false interaction among the variety of domains.

\subsection{Target Identification And Drug Release By Nanorobots}

According to earlier period experienced researchers have attempted in order to increase methods that effort on the affected area that are measured as injured cells leaving the strong cells undamaged Angioplasty surgery is assumed to be out behind the times in which even the close by cells also get injured and this leads to high probability of further multiply of injured cells[9].It leads to the development of Nanorobots .Nanorobots via chemical sensors as embedded nanoelectronics can be planned to sense diverse levels of alpha-NAGA signals . Fig 5 depicts use of Nanorobots in biohazard defence system.

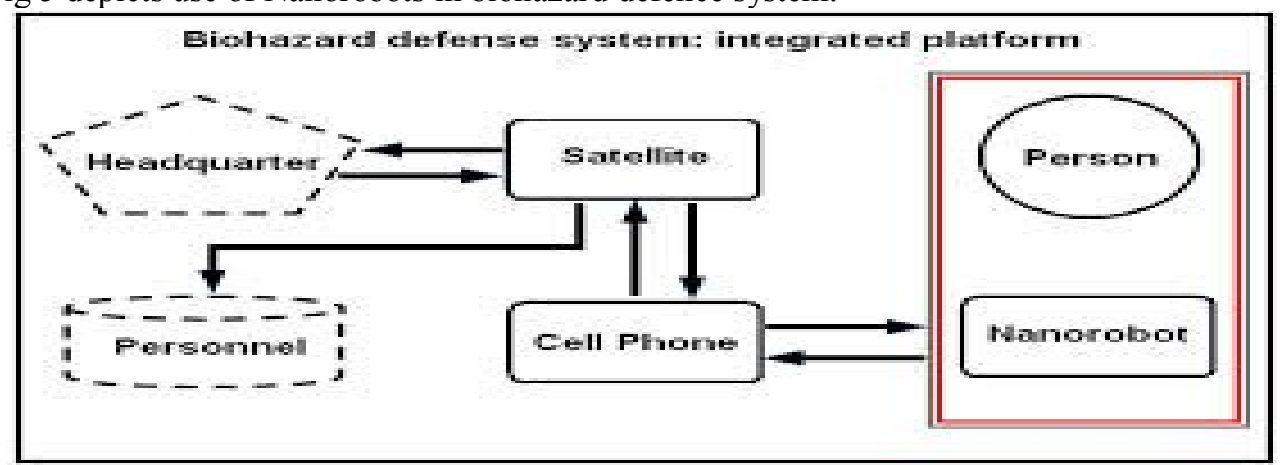

Fig 5. Biohazard defence system.

Nanorobots could be employed as movable medical devices inserted in the course of the bloodstream are used. The activities utilized by influenza to cell attack and union are almost related with strategy that are used by other viruses such as Smallpox or SARS. The planned proposal with Nanorobot model is quite efficient design that can be applied to influenza prediction can bring a change in biohazard defence possibilities, thus implementing an innovative virus fighting technology.

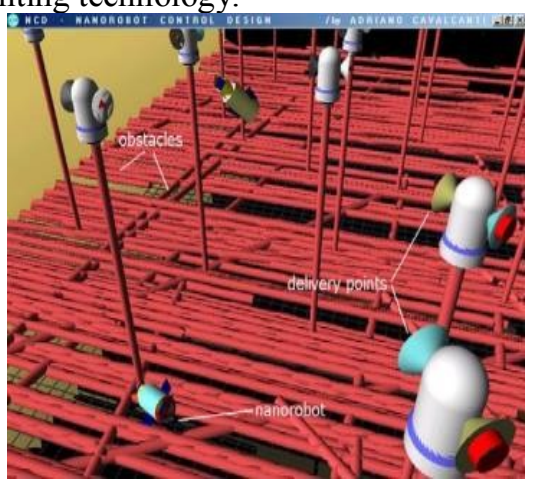

Fig 5.1 Nanorobots for drug release. 
There are many changes inside our body which help the Nanorobots to detect the target and supply the drug at a specific location. This is depicted in the Fig 5.1. Recently many techniques have been developed in order to spot the target that is based on the biological chemical sensors produced within our body. Lock and Key mechanism has been employed in order to supply the definite drug to the definite cells only. The Nanorobots that carries specific drug is treated as lock that supplies to the particular affected cell called as key in order to detect the target [10].

\subsection{Bypass Surgery}

People with coronary artery disease are treated by doing heart bypass surgery. This is done to increase the flow of blood to the heart muscles. More than one artery may be bypassed during the operation but this method is not without side effects. Instead a Nanorobot can be used to operate. This Nanorobot contains two regions an outer region and an inner region. This Nanorobot is attached with an electric motor for its circulation. Along with the electric motor an artery thermometer, a microprocessor, a camera and a revolving needle is incorporated into the Nanorobot. The microprocessor will control the complete operation [11]. A radioactive material is infused into the outer region, which will help to trace the movements of the Nanorobot (fig 4). The Nanorobot can be switched anytime on and off anytime using a magnetic switch. The Nanorobot can be injected into femoral artery in the leg which is one of the largest arteries in the body.

The induced Nanorobot with the help of the diamond chip can cut out the plaque and grind them into micro particles. The Nanorobot is removed by directing the Nanorobot to anchor to a blood vessel that is easily accessible from outside.

\subsection{Treatment Of Arteriosclerosis}

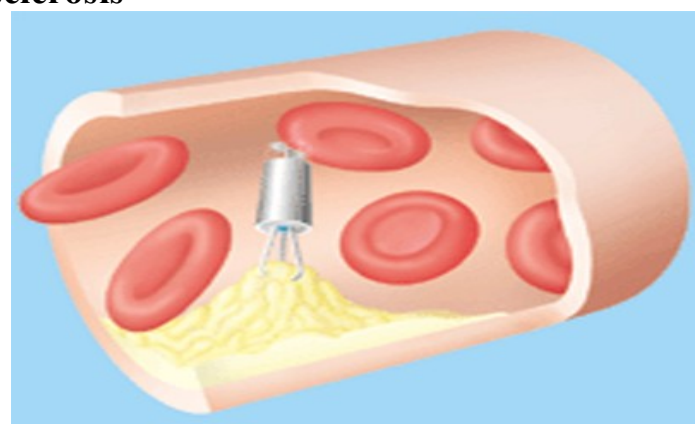

Fig.6 Nanorobots clearing plaque.

Arteriosclerosis occurs when plaque builds along the walls of the arteries which will block the blood flow through them. Nanorobots could be used to cut away all the plaques and clear the arteries and capillaries and hence reducing the chances of heart attack [12].

\subsection{Demylination}

A demyelinating disease is a disease of the nervous system where the myelin sheath of neurons is injured [13].Transference of signals alongside the nerve will be damaged or misplaced thus nerve ultimately shrinks.Nanorobot will be situated on nerves which with the nerve impulses and choose on the path it will acquire. The Nanorobots locate this demyelinised area and bring myelin sheath on it .Hence it is doable to make sure that disease could be treated.Nanorobot which is located on nerves will detect electrical signals (nerve impulses and obtain conclusion on the desired path to be taken).A set of Nanorobot can distribute an independent, precise and least enveloping surgical method to treat demyelination should be made.

\section{Current Status}

Currently, nanotechnology presents a broad platform in the field of cardiovascular science by contributing tools to explore the frontiers of cardiac science at the basic cellular level. Fullerene compounds made up of nanomaterials may be served as antiviral agents. The global market for nanoscale devices and molecular form may arise up to 28\% a year, increasing from \$406 million in 2002 to \$1.37 billion in 2007, with a $35 \% /$ year expansion pace in revenues from biomedical nanoscale devices [14].

\section{Challenges In Medical Application}

The Nanorobots are undetectable to unprotected eye that make them tough to control and work [15].Some doctors worry that the nanoparticles are so small, that they could easily cross the blood-brain barrier, a membrane that protects the brain from harmful chemicals in the bloodstream. The grand challenge to develop the tools to properly assess risk to human health and to the environment. 


\section{Conclusion}

By end of $21^{\text {th }}$ century use of Nanorobots in medical field will eradicate all common diseases, sufferings. Nanotechnology in medical field is a quite revolutionary and stimulating technique for a good quality health care.With the application to healthcare, nanotechnology is indeed quite the stimulating and revolutionary technique in the pursuit of quality healthcare. Nanomedicine used to advance human health using molecular tools and nanoparticles.

Finally, and most importantly, no actual working Nanorobot has yet been built. Several theoretical designs have been proposed that look good on paper, but these preliminary designs could change significantly after necessary research, development and testing has been completed.

\section{References}

[1]. Cavalcanti A, smithJ.A.Claytn, Nanorobots for laparoscopic cancer surgery,International Conference on IEEE/ACIS,2007

[2]. Adriano Cavalcanti, Bijan Shirinzadeh, Tad Hogg, Luis C. Kretly: CMOS-based Nanorobot to Combat Cancer CAN Centre for Automation in Nano biotech Sao Paulo SP 01540, Brazil

[3]. Venkatesan M, D.Y Patil and Jolad B. Nanorobots in cancer treatment, IEEEInternational conference 2010 , pp.258 to 264

[4]. Deepa.R.Parmar, Julee.P.soni and Apexa.D. Patel and Dhrubo Jyoti Sen, Nanorobotics in advances in Pharamaceutical sciences, International Journal of Drug Development \& Research.Vol.2, Issue2I, june2010

[5]. Neetha J.Shetty, P.Swati and K.David,Nanorobots Future in dentistry, The Saudi Dental Journal v.25 (2) April 2013

[6]. Ana-Maria Dumitrescu,andCristina D a s c a lu, Dental Nanorobots small instruments with large potential, Romanian journal of oral rehabilitation., Vol.3,No.4,December2011

[7]. Ashley Feinberg, DNA Nanobots Turn Cockroaches into Living, 8-Bit Computers, April 08, 2014

[8]. Shawn Douglas, DNA Nanorobot to Trigger Targeted Therapeutic Responses, Harvard's Wyss Institute 2012

[9]. Sarah Spickernel, DNA nanorobots deliver drugs in living cockroaches,Nature Nanotechnology Journal, April 2014

[10]. Adriano Cavalcanti, Bijan Shirinzadeh, Mingjun Zhang and Luiz C.Kretly, Nanorobot Hardware Architecture for Medical Defense Sensors, 2932-2958DOI: 10.3390/s8052932 May 2008

[11]. Shinob M.C and Jidhin G, Replacement of Heart Bypass Surgery by Nanorobots, International Journal of Advance Research and technology,

[12]. Drexler KE, The Coming Era of Nanotechnology, Engines of Creation, Anchor Press, New York, 1986 updated in 2007

[13]. S.M.Masudur Rahman Al-Arif, Niamey Quaver, Al Mamun Shaon, Dr. Kazi Khairul Islam, and Sensor based Autonomous Medical Nanorobots: A cure to demyelination, Journal of Selected Areas in Nanotechnology (JSAN), September. 2011.

[14]. Nathan.A.Weir, Dannele.p.Sierra,and James.f.Jones, A Review of Research in the Field of Nanorobotics,Sandia National Laboratories,New Mexico

[15]. Robert A freitas Jr, Curent status of Nanomedicine and Medical nanorobotics, Journalof computational and theoretical Nanoscience, Vol 2,Jan 2005 